\title{
ANÁLISIS EXPERIMENTAL DE LAS BARRAS DE MADERA LAMINADA CON SECCIÓN TUBULAR UTILIZADAS EN LA CONSTRUCCIÓN DE UNA MALLA ESPACIAL
}

\author{
EXPERIMENTAL ANALYSIS OF GLUED-LAMINATED TIMBER BARS \\ WITH BOX SECTION USED IN THE CONSTRUCTION OF A SPATIAL GRID
}

\author{
J. Estévez Cimadevila ^, D. Otero Chans ${ }^{1}$ E. Martín Gutiérrez ${ }^{1}$, J. Vázquez Rodríguez ${ }^{1}$
}

\begin{abstract}
RESUMEN
La construcción de una malla espacial empleando módulos apilables ejecutados con barras de madera laminada de sección tubular ha constituido el germen para abordar una campaña experimental del comportamiento de este tipo de piezas. El artículo presenta los resultados del trabajo experimental realizado, tanto con anterioridad a la construcción de la estructura, como los ensayos posteriores sobre barras reales reservadas durante la ejecución de la obra. Se han realizado ensayos tanto de tracción como de compresión empleando barras con diferentes esbelteces. Los resultados ponen de manifiesto el interés de las soluciones propuestas y la viabilidad de su empleo en esta tipología estructural.
\end{abstract}

Palabras clave: Estructuras de madera, barras de madera laminada de sección tubular o sección cajón, ensayos destructivos.

\begin{abstract}
The construction of a spatial grid using stackable modules of glued-laminated timber bars with box section has provided the germ for an experimental approach of the behaviour of these types of bars. The paper presents the results of experimental campaign, both prior to the construction of the structure, such as real bars subsequent trials. Both tension tests and compression tests using bars with different slenderness have been conducted. The results show the interest of the proposed solutions and the feasibility of its use in this type of structure.
\end{abstract}

Keywords: Wood structures, glued-laminated timber box elements, destructive testing.

\section{INTRODUCCIÓN}

Las mallas espaciales constituyen una de las tipologías estructurales más significativas del siglo XX. La infinidad de construcciones realizadas en los más de 100 años transcurridos desde la aparición de los primeros prototipos ponen de manifiesto su adecuación y eficiencia para cubrir grandes espacios con una solución de gran ligereza. 
Por otra parte, la madera laminada, cuya aparición puede datarse en 1906 con la patente de Otto Hetzer, constituye un material de grandes prestaciones mecánicas y reducido peso específico, lo que lo convierte en un material idóneo para su utilización en estructuras de cubierta de elevada luz.

A pesar de la casi total coincidencia en el tiempo del despegue tecnológico de la madera como material y de la malla espacial como tipología estructural, resulta sorprendente la escasísima existencia de ejemplos de su utilización conjunta. Tuvieron que transcurrir 80 años hasta que se materializaron las primeras propuestas de mallas espaciales de doble capa utilizando barras de madera (Huybers 1986). Dentro de este panorama destacan dos obras singulares: el puente sobre el río Isar en Thalkirchen (Alemania) diseñado por el ingeniero Richard J. Dietrich (Figura 1) y, especialmente, la cubierta del Oguni Dome en Kyushu (Japón) proyectada por el arquitecto Hamura Shoei Yoh (Figura 2).

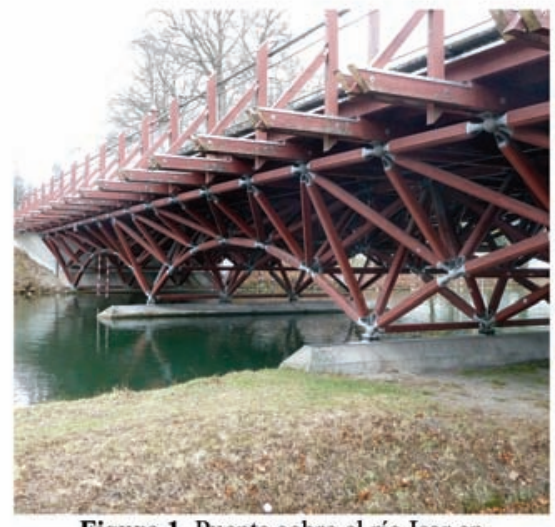

Figura 1. Puente sobre el río Isar en Thalkirchen. Alemania. (Dietrich 1993).

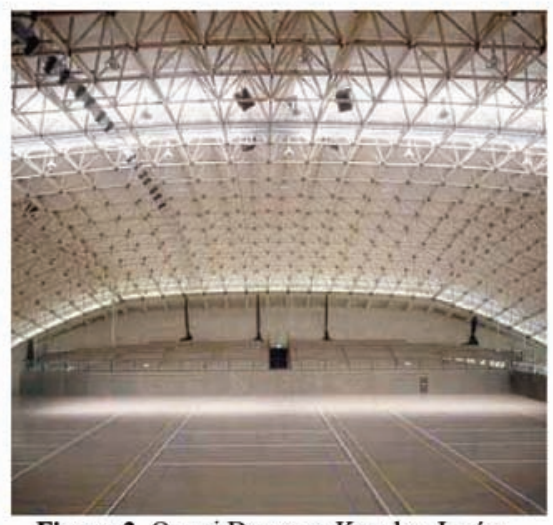

Figura 2. Oguni Dome en Kyushu. Japón. (Shoei 1988).

Por encargo de la Universidad de A Coruña, los arquitectos J. Estévez y J. Vázquez redactan en junio de 2001 el proyecto de un edificio destinado a uso deportivo (Figura 3). Se trata de una edificación singular constituida por paredes portantes de superficie reglada de un pié de fábrica de ladrillo, y una estructura de cubierta de malla espacial de doble capa con barras de madera (Estévez y Vázquez 2001).

Además de la singularidad de los muros cerámicos, deudores de las maravillosas estructuras de Eladio Dieste, la principal aportación de la construcción realizada se centra en dos aspectos de la estructura de cubierta: la apilabilidad de los módulos constitutivos de la malla espacial (Figura 4), y el empleo de piezas de madera de sección tubular (Figura 5). El carácter apilable de los módulos de la malla permite su ejecución en taller facilitando su montaje, almacenamiento y transporte a la obra. Por su parte, la utilización de secciones huecas da lugar a barras de muy reducido peso y gran eficiencia estructural frente a las solicitaciones de compresión. La estructura de madera en la obra señalada ha supuesto una repercusión de $8.98 \mathrm{~kg} / \mathrm{m}^{2}$, que se incrementa hasta $14.43 \mathrm{~kg} / \mathrm{m}^{2}$ si consideramos los herrajes metálicos que conforman las uniones. Las únicas propuestas de mallas espaciales existentes con barras de sección tubular se circunscriben a algunos prototipos ensayados en laboratorio utilizando bamboo (Ghavami y Moreira 1993) y a las recientes estructuras espaciales con tubos de cartón construidas por el arquitecto japonés Hamura Shigeru Ban. 


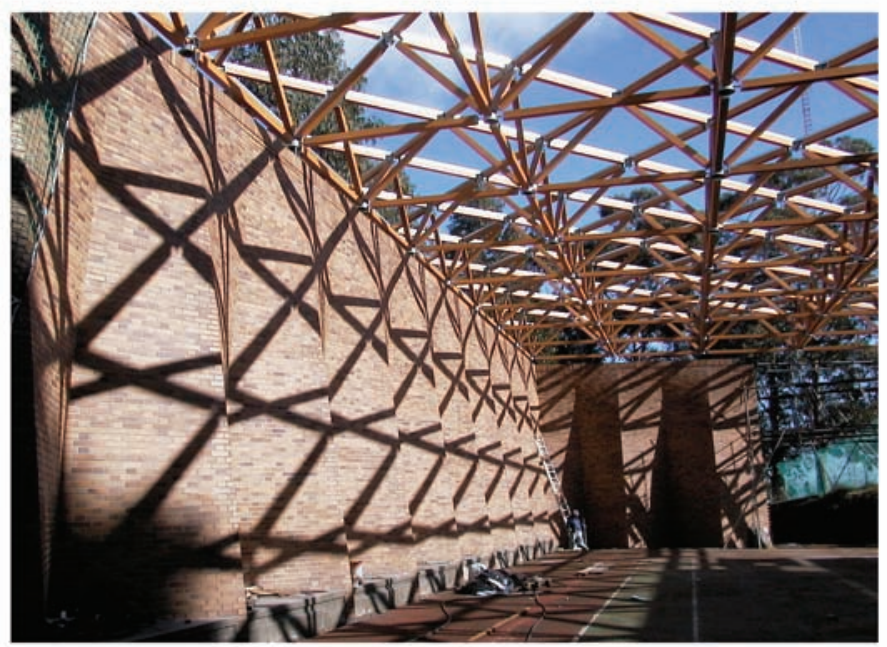

Figura 3. Edificio deportivo de la Universidad de A Coruña. España.

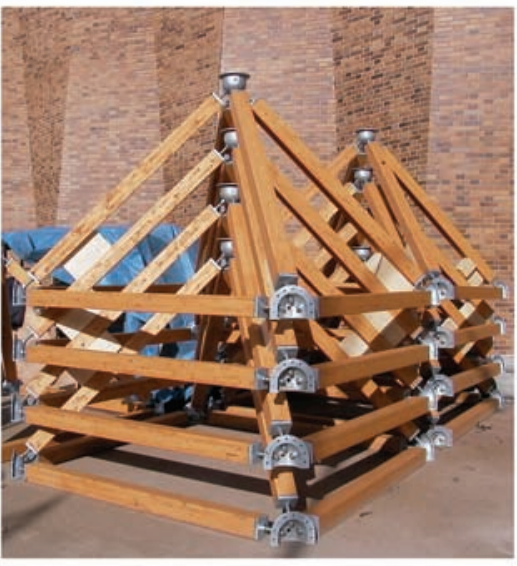

Figura 4. Módulos apilados.

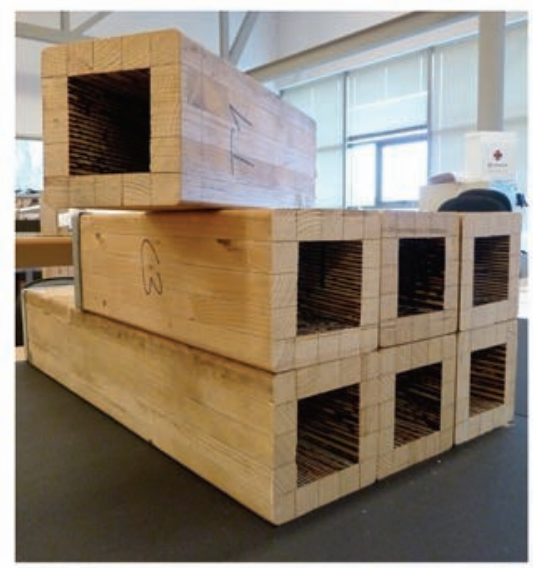

Figura 5. Barras de sección hueca.

El principal problema que se plantea en la construcción de una malla espacial empleando barras de madera consiste en el diseño de un eficaz enlace de las barras al nudo. Este problema adquiere especial importancia cuando las barras son de sección tubular y se encuentran solicitadas a tracción. Todas las soluciones planteadas se basaron en tapar los extremos de las barras. Esta solución, empleando tacos extremos de madera de alta densidad, presentaba varias ventajas: en primer lugar, permitía resolver el problema que se plantearía en caso de incendio si las llamas pudiesen penetrar al interior de la barra; en segundo lugar, el disponer de una madera de alta densidad en las cabezas facilitaba el anclaje de barras roscadas encoladas con resina epoxi, con las que se podía realizar fácilmente la unión con el nudo metálico; finalmente, los propios tacos actuaban como plantilla para asegurar una correcta ejecución de la geometría de las piezas tubulares. 
Dada la ausencia de datos en la bibliografía técnica respecto al comportamiento de este tipo de barras, se abordó una doble campaña de ensayos con objeto de verificar la viabilidad de la solución propuesta. La primera campaña se realizó sobre probetas previamente a la construcción de la estructura. La segunda campaña se realizó, transcurridos 10 años desde la construcción de la estructura, sobre 21 barras reales que se reservaron durante la ejecución de la obra, con objeto de conocer también la posible afectación en su comportamiento del envejecimiento de los materiales. Los resultados de ambos ensayos constituyen el objeto del presente artículo.

\section{MATERIALES Y MÉTODO}

\section{Ensayos previos a la construcción de la estructura}

Los primeros ensayos experimentales realizados no constituyeron una campaña experimental propiamente dicha, sino un conjunto de pruebas de diferentes alternativas de diseño y constructivas que tenían por finalidad dar viabilidad al proyecto realizado teniendo presente el limitado presupuesto de la obra y el tiempo disponible para la redacción del proyecto.

Las probetas se confeccionaron con madera de Picea abies y tacos extremos de elondo (Erythrophleum ivorense). El diseño y las características geométricas de las probetas ensayadas a tracción con la solución final de extremo de barra pueden observarse en la figura 6. Se aprecia que la conexión entre la madera conífera de la barra y el taco extremo de madera frondosa se realiza rebajando el espesor de la pared del tubo de madera con objeto de incrementar la superficie de contacto entre ambas maderas (conífera y frondosa). El encolado entre sí de estas dos piezas se realizó con adhesivo de resorcina-formaldehido. El número de probetas ensayadas con este diseño ha sido de 8. Los ensayos se realizaron en el Laboratorio de Construcción del Centro de Innovación Tecnológica en Edificación e Ingeniería Civil de la Universidad de A Coruña. Cuando se realizaron los ensayos la máquina universal disponible INSTRON 8805 carecía de mordazas de tracción, por lo que los ensayos se realizaron empleando un pórtico metálico y un gato hidráulico que aplicaba la tracción por reacción contra la losa de carga, siguiendo el sistema que se muestra en las figuras 7 y 8 .

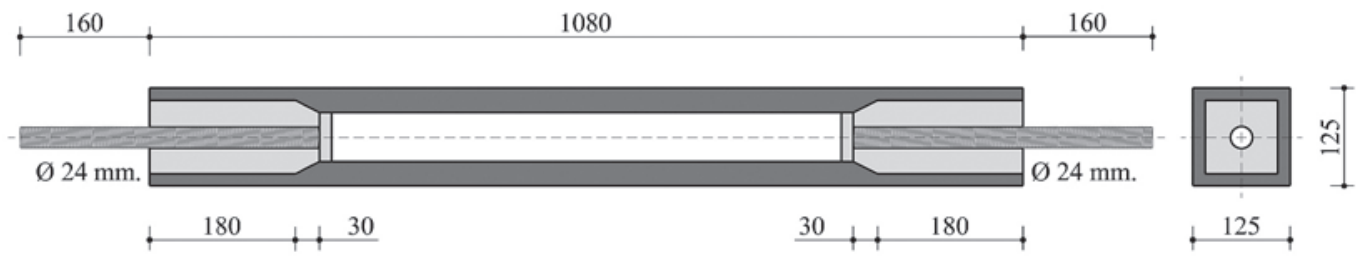

Figura 6. Características geométricas de las probetas ensayadas. Dimensiones en mm. 


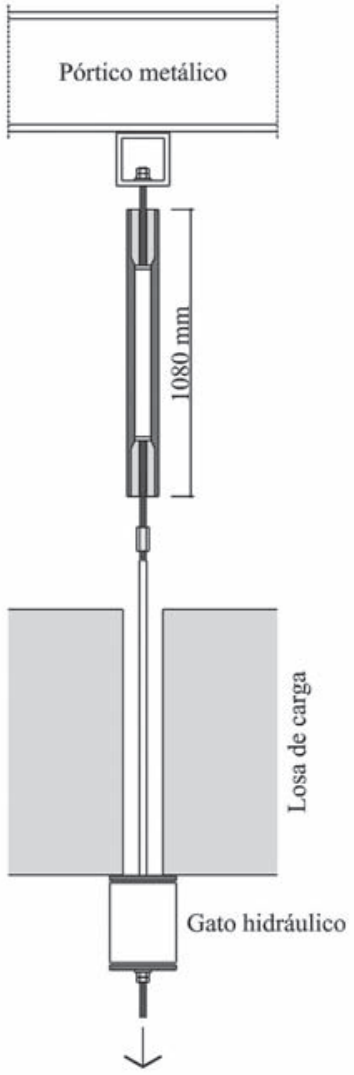

Figura 7. Esquema del dispositivo de ensayo.

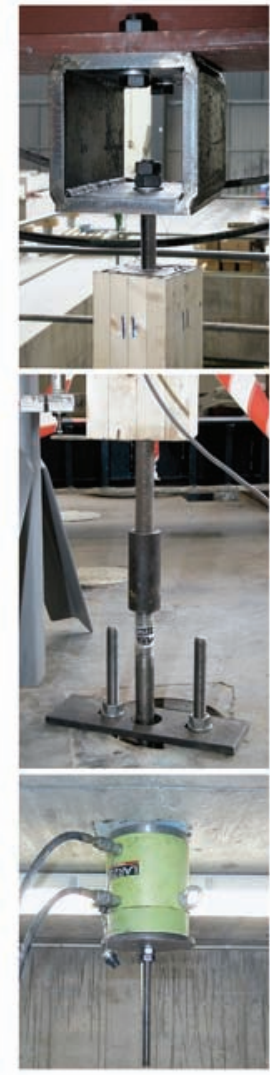

Figura 8. Imágenes del dispositivo de ensayo.

\section{Campaña de ensayos con barras de dimensión real}

Como ya se ha señalado, durante la ejecución de la estructura se reservaron una serie de barras para su posterior ensayo. En total se guardaron 17 barras de $2450 \mathrm{~mm}$ de longitud a ejes de nudos de la estructura y 4 barras de $3465 \mathrm{~mm}$, todas ellas con una sección transversal nominal de 125x125 mm y un espesor de $25 \mathrm{~mm}$. Las barras se confeccionaron en madera laminada de Picea abies, clase resistente GL28h. Las barras se cerraron en sus extremos con unas piezas de $75 \times 75 \times 250 \mathrm{~mm}$ de madera frondosa de elondo (Erythrophleum ivorense). Para facilitar el encolado de las barras roscadas en el taco de elondo, se subdividió dicho taco en dos piezas de $37.5 \times 75 \times 250 \mathrm{~mm}$. El encolado entre sí de estas dos piezas así como con las paredes interiores de las barras de madera laminada se realizó con adhesivo de resorcina-formaldehido. Para la conexión con los nudos de acero fundido con forma de casquete esférico de la malla espacial, se dispusieron barras roscadas de $24 \mathrm{~mm}$ de diámetro de acero de calidad 8.8, con un valor de tensión de rotura $\mathrm{f}_{\mathrm{u}}=800 \mathrm{~N} / \mathrm{mm}^{2}$ y un límite elástico $\mathrm{f}_{\mathrm{y}}=640 \mathrm{~N} / \mathrm{mm}^{2}$. La unión de las barras roscadas a la pieza de elondo se realizó con resina epoxi HILTI HIT-RE 500, empleando un espesor de adhesivo de $1 \mathrm{~mm}$.

El proceso de fabricación de las barras huecas fue sumamente sencillo. Las caras que conforman la pieza tubular se obtuvieron directamente por simple corte de piezas de madera laminada de sección maciza, utilizando secciones con un ancho igual a 4 veces el espesor de pared de la pieza tubular. Evidentemente, también podrían haberse utilizado para las caras tableros LVL en lugar de la madera laminada, lo que sin duda constituye una alternativa de gran interés para la ejecución de piezas tubulares. 
El encolado de las piezas se realizó en dos fases. En una primera fase se encolaron las caras opuestas de cada tubo utilizando como plantilla los tacos extremos de anclaje y una o dos piezas intermedias a modo de separadores. En la segunda fase, se encolaron las otras dos caras quedando así conformada cada pieza tubular. Tal y como se muestra en la figura 9, se pueden encolar numerosas barras simultáneamente, siendo adecuadas para el prensado de las superficies encoladas las prensas horizontales habitualmente utilizadas en la fabricación de secciones macizas de madera laminada.

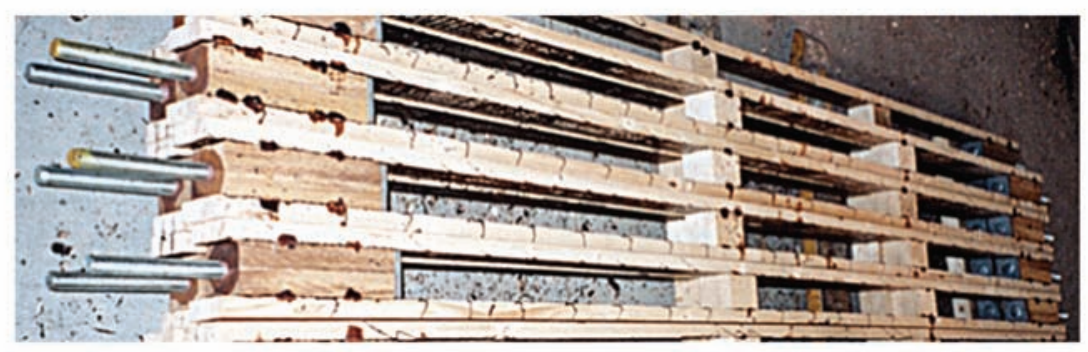

Figura 9. Ejecución de las barras huecas de $3465 \mathrm{~mm}$ de longitud.

Las barras estuvieron almacenadas en el propio Departamento de Tecnología de la Construcción durante 10 años desde su fabricación. Su almacenaje se realizó en un ambiente interior; no obstante, no se adoptó ningún tipo especial de protección, de forma que estuvieron expuestas a radiación solar directa durante los períodos estivales.

Para la campaña de ensayos se dividió el número total (17) de probetas de $2450 \mathrm{~mm}$ en dos lotes: uno de 9 probetas para ensayar a doble tracción y otro de 8 probetas para ensayar a doble compresión.

Todas las barras de mayor longitud $(3465 \mathrm{~mm})$ se ensayaron a doble compresión. Entendimos que sería suficiente realizar el ensayo de tracción sólo sobre las barras de $2450 \mathrm{~mm}$ puesto que la tracción es estabilizante de la forma y, por tanto, la longitud de las barras no afecta a su capacidad de carga. Por otra parte, el ensayo a compresión de las barras largas podía aportar datos interesantes en relación con la incidencia del fenómeno del pandeo en este tipo de piezas cuando la esbeltez alcanza valores apreciables.

Finalmente, en las zonas no dañadas de las barras ensayadas a tracción se extrajeron unas rebanadas de $225 \mathrm{~mm}$ de longitud para su ensayo a compresión simple prescindiendo de la incidencia de la flexión lateral por pandeo. Ello permitió completar los ensayos de compresión disponiendo de los valores de carga última para tres longitudes $(225,2450$ y $3465 \mathrm{~mm})$ y, consecuentemente, tres rangos de esbeltez.

Durante los ensayos se procedió también a determinar el módulo de elasticidad tanto a tracción como a compresión. A tal efecto se midió la deformación en el tramo central de las barras disponiendo dos extensómetros con el objeto de minimizar posibles efectos de distorsión. Se emplearon extensómetros Schreiber, SM 222.20.2.S con un rango de $-10 \mathrm{~mm} \mathrm{a}+10 \mathrm{~mm}$.

Antes de proceder a los ensayos se midió la humedad de las piezas a ensayar, lo que arrojó una humedad para el lote de 9.9\%. En base a ello, se decidió almacenar la totalidad de las barras en una cámara de acondicionamiento en una atmósfera normalizada a $20^{\circ} \mathrm{C}$ y $65 \%$ de humedad relativa hasta alcanzar la humedad de equilibrio correspondiente (12\%). Se ha utilizado una estufa de secado Raypa, DAF-635. La determinación de la humedad se ha realizado siguiendo el criterio de la normativa UNEEN 13183-1 (2002) que determina su valor por diferencia de pesadas. 
Los ensayos se realizaron en el Laboratorio de la Escuela Politécnica Superior de Lugo utilizando un banco de ensayos multieje con una célula de carga Micro Test, PB2-F/600, con una capacidad de 600 $\mathrm{kN}$. Los ensayos se realizaron ajustándose a las especificaciones de la normativa UNE-EN 408 (2011). En la figura 10 se muestra el ensayo a compresión correspondiente a una de las barras de $3465 \mathrm{~mm}$ de longitud. La figura 11 recoge el dispositivo colocado para la determinación del módulo de elasticidad.

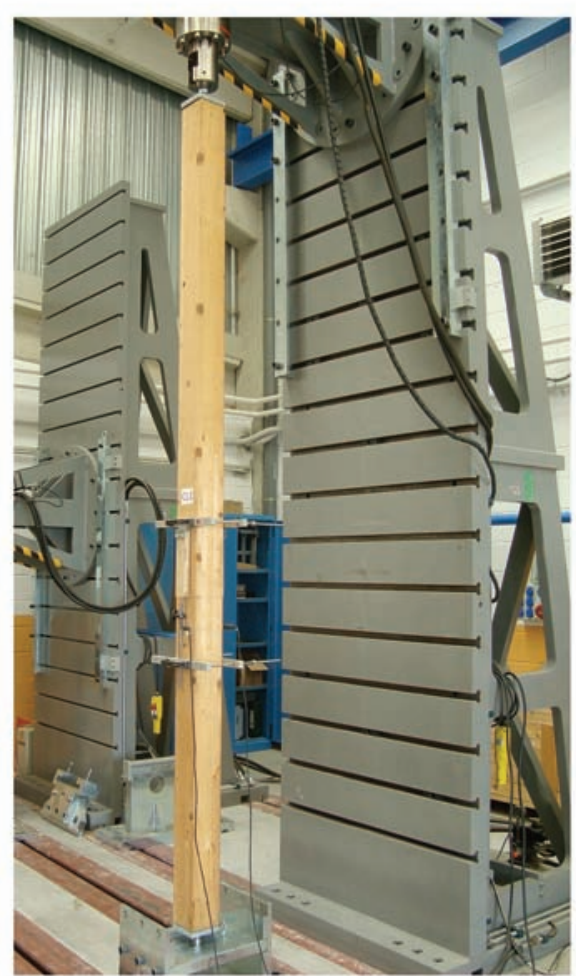

Figura 10. Dispositivo para el ensayo de compresión.

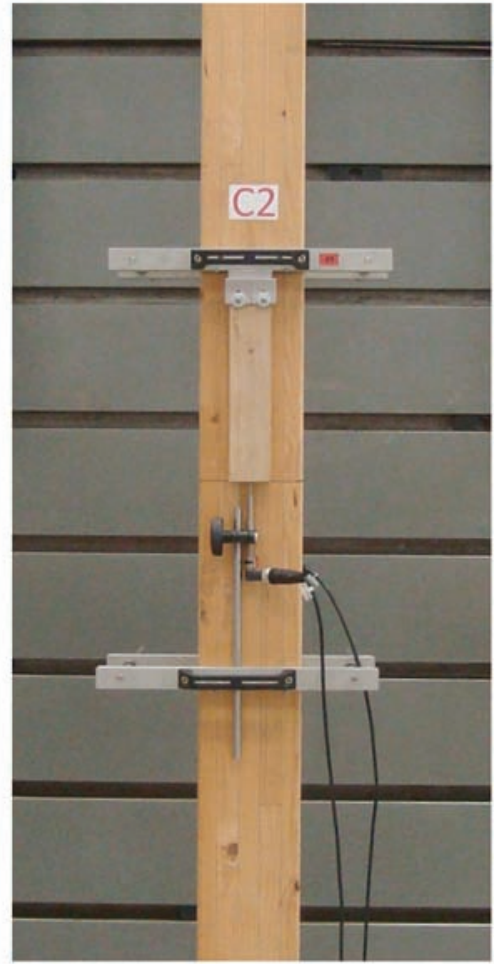

Figura 11. Dispositivo para la obtención del módulo de elasticidad. 


\section{RESULTADOS}

\section{Ensayos previos a la construcción de la estructura}

Los resultados obtenidos en el ensayo de tracción se recogen en la tabla 1. Se indica para cada uno de los especímenes ensayados el valor de carga última, la tensión paralela a la fibra y la tensión tangencial promedio en la superficie de contacto entre maderas. También se muestran los valores correspondientes al $5 \%$ percentil.

El fallo se alcanzó, en todos los casos, por la rotura del plano de contacto del adhesivo con la madera laminada de la barra hueca (Figura 12).

Tabla 1. Resultados de los ensayos previos a la construcción de la estructura

\begin{tabular}{|c|c|c|c|c|c|c|}
\hline & Result & dos de los & sayos & Valore & la serie $\left(5^{\circ}\right.$ & ercentil) \\
\hline Probeta & $\begin{array}{l}\text { Carga última } \\
(\mathrm{kN})\end{array}$ & $\begin{array}{l}\text { Tensión } \\
\text { paralela } \\
\text { a la fibra } \\
\left(\mathrm{N} / \mathrm{mm}^{2}\right)\end{array}$ & $\begin{array}{c}\text { Tensión } \\
\text { tangencial } \\
\text { promedio } \\
\left(\mathrm{N} / \mathrm{mm}^{2}\right)\end{array}$ & $\begin{array}{c}\text { Carga } \\
\text { última } \\
(\mathrm{kN})\end{array}$ & $\begin{array}{l}\text { Tensión } \\
\text { paralela } \\
\text { a la fibra } \\
\left(\mathrm{N} / \mathrm{mm}^{2}\right)\end{array}$ & $\begin{array}{c}\text { Tensión } \\
\text { tangencial } \\
\text { promedio } \\
\left(\mathrm{N} / \mathrm{mm}^{2}\right)\end{array}$ \\
\hline 7 & 183.91 & 17.03 & 2.46 & \multirow{8}{*}{158.75} & \multirow{8}{*}{14.70} & \multirow{8}{*}{2.12} \\
\hline 8 & 189.00 & 17.50 & 2.52 & & & \\
\hline 9 & 160.82 & 14.89 & 2.15 & & & \\
\hline 10 & 194.52 & 18.01 & 2.60 & & & \\
\hline 11 & 182.35 & 16.88 & 2.44 & & & \\
\hline 12 & 186.27 & 17.25 & 2.49 & & & \\
\hline 13 & 157.63 & 14.60 & 2.11 & & & \\
\hline 14 & 180.03 & 16.67 & 2.40 & & & \\
\hline
\end{tabular}
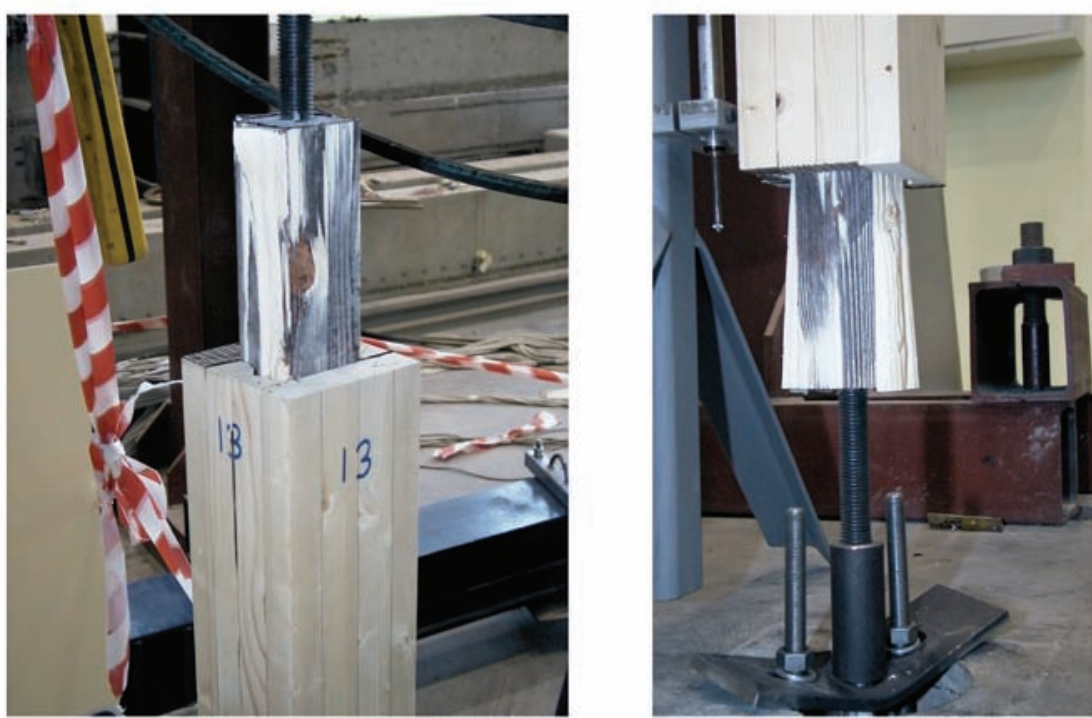

Figura 12. Ejemplos representativos del fallo en el ensayo de tracción. 


\section{Ensayos de barras reales}

La tabla 2 detalla los resultados obtenidos en el ensayo de tracción. Se indican la carga última, el módulo de elasticidad y el valor de tensión paralela a la fibra para cada uno de los especímenes ensayados, así como los valores correspondientes al 5\% percentil.

Tabla 2. Resultados de los ensayos de barras reales de tracción.

\begin{tabular}{|c|c|c|c|c|c|c|c|c|}
\hline & \multicolumn{4}{|c|}{ Resultados de los ensayos } & \multicolumn{4}{|c|}{ Valores de la serie ( $5 \%$ percentil) } \\
\hline Probeta & $\begin{array}{l}\text { Carga } \\
\text { última }\end{array}$ & $\begin{array}{l}\text { Módulo de } \\
\text { elasticidad }\end{array}$ & $\begin{array}{l}\text { Tensión } \\
\text { paralela }\end{array}$ & $\begin{array}{c}\text { Tensión } \\
\text { tangencial }\end{array}$ & $\begin{array}{l}\text { Carga } \\
\text { última }\end{array}$ & $\begin{array}{l}\text { Módulo de } \\
\text { elasticidad }\end{array}$ & $\begin{array}{l}\text { Tensión } \\
\text { paralela }\end{array}$ & $\begin{array}{c}\text { Tensión } \\
\text { tangencial }\end{array}$ \\
\hline & $(\mathrm{kN})$ & $\left(\mathrm{N} / \mathrm{mm}^{2}\right)$ & $\begin{array}{l}\text { a la fibra } \\
\left(\mathrm{N} / \mathrm{mm}^{2}\right)\end{array}$ & $\begin{array}{l}\text { promedio } \\
\left(\mathrm{N} / \mathrm{mm}^{2}\right)\end{array}$ & $(\mathrm{kN})$ & $\left(\mathrm{N} / \mathrm{mm}^{2}\right)$ & $\begin{array}{l}\text { a la fibra } \\
\left(\mathrm{N} / \mathrm{mm}^{2}\right)\end{array}$ & $\begin{array}{c}\text { promedio } \\
\left(\mathrm{N} / \mathrm{mm}^{2}\right)\end{array}$ \\
\hline T1 & 150.72 & 11134 & 15.18 & 2.03 & \multirow{9}{*}{$\begin{array}{l}130.55 \\
151.22^{*}\end{array}$} & \multirow{9}{*}{11148} & \multirow{9}{*}{$\begin{array}{l}13.48 \\
15.27^{*}\end{array}$} & \multirow{9}{*}{$\begin{array}{l}1.76 \\
2.04^{*}\end{array}$} \\
\hline T2 & 117.10 & 12382 & 12.34 & 1.58 & & & & \\
\hline T3 & 155.95 & 12760 & 15.87 & 2.12 & & & & \\
\hline $\mathrm{T} 4$ & 175.63 & 11701 & 17.84 & 2.38 & & & & \\
\hline T5 & 174.61 & 11251 & 17.65 & 2.37 & & & & \\
\hline T6 & 160.16 & 11540 & 16.19 & 2.17 & & & & \\
\hline $\mathrm{T7}$ & 158.84 & 12563 & 16.21 & 2.15 & & & & \\
\hline T8 & 152.15 & 11814 & 15.43 & 2.06 & & & & \\
\hline T9 & 161.68 & 11168 & 16.39 & 2.19 & & & & \\
\hline
\end{tabular}

(* resultados prescindiendo de probeta T2)

La figura 13 muestra un ejemplo representativo del tipo de rotura observada. Todas las barras, con excepción de la pieza T2, alcanzaron la rotura por esfuerzo cortante de la madera laminada de Picea abies en el plano de contacto con el adhesivo. También se ha observado que la rotura va siempre acompañada de la hienda de la pieza tubular. En el caso del ejemplar T2, cuyo valor de carga última ha sido significativamente menor, se ha observado el fallo en la línea de encolado de las dos piezas en que se subdividió el taco de elondo, donde se ha apreciado una clara deficiencia en la ejecución del encolado de la unión, lo que explica la reducción en su valor de carga última. Esta es la razón por lo que en los valores de carga última y tensión de la serie, reflejados en la tabla 2 , se han indicado también los resultados obtenidos prescindiendo de dicha probeta $\mathrm{T} 2$ por los defectos detectados en la ejecución de la misma. 


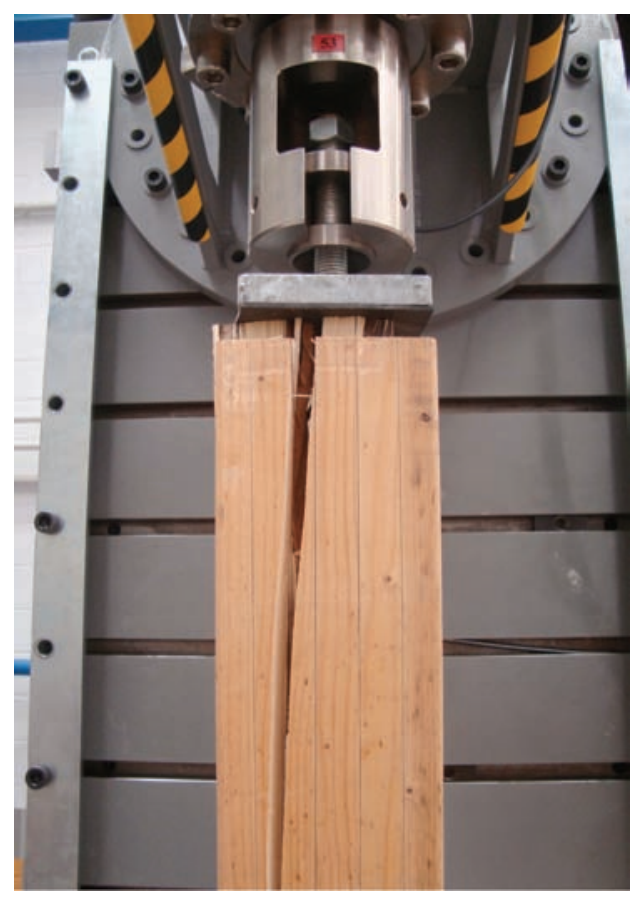

Figura 13. Forma de fallo característica de las barras sometidas a tracción.

La tabla 3 detalla los resultados de todas las piezas ensayadas a compresión. Las piezas de $225 \mathrm{~mm}$ se ensayaron a compresión simple con el sistema habitual de aplicar carga a través de un plato. En el caso de las barras largas, para tener en cuenta el efecto del pandeo, se dispuso un sistema de rótulas esféricas en ambos extremos de forma que se garantizase una completa libertad de giro. Los valores de longitud de las barras indicados en la tabla corresponden a la distancia entre puntos de localización de las citadas rótulas esféricas, pues dicha dimensión es la considerada a los efectos de determinación del valor de esbeltez. 
Tabla 3. Resultados de los ensayos de compresión

\begin{tabular}{|c|c|c|c|c|c|c|c|}
\hline \multirow[b]{2}{*}{$\begin{array}{l}\text { Longitud } \\
\text { probeta } \\
(\mathrm{mm})\end{array}$} & \multirow[b]{2}{*}{ Probeta } & \multicolumn{3}{|c|}{ Resultados de los ensayos } & \multicolumn{3}{|c|}{ Valores de la serie ( $5 \%$ percentil) } \\
\hline & & $\begin{array}{c}\text { Carga } \\
\text { última } \\
(\mathrm{kN})\end{array}$ & $\begin{array}{l}\text { Módulo de } \\
\text { elasticidad } \\
\left(\mathrm{N} / \mathrm{mm}^{2}\right)\end{array}$ & $\begin{array}{l}\text { Tensión } \\
\text { paralela } \\
\text { a la fibra } \\
\left(\mathrm{N} / \mathrm{mm}^{2}\right)\end{array}$ & $\begin{array}{c}\text { Carga } \\
\text { última } \\
(\mathrm{kN})\end{array}$ & $\begin{array}{l}\text { Módulo de } \\
\text { elasticidad } \\
\left(\mathrm{N} / \mathrm{mm}^{2}\right)\end{array}$ & $\begin{array}{l}\text { Tensión } \\
\text { paralela } \\
\text { a la fibra } \\
\left(\mathrm{N} / \mathrm{mm}^{2}\right)\end{array}$ \\
\hline \multirow{5}{*}{225} & T1 & 386.51 & & 38.93 & \multirow{5}{*}{361.63} & & \multirow{5}{*}{36.58} \\
\hline & $\mathrm{T} 2$ & 401.61 & & 42.32 & & & \\
\hline & $\mathrm{T} 3$ & 411.05 & & 41.82 & & & \\
\hline & $\mathrm{T} 4$ & 366.26 & & 37.20 & & & \\
\hline & T5 & 360.47 & & 36.43 & & & \\
\hline \multirow{8}{*}{2050} & $\mathrm{Cl}$ & 293.09 & 12048 & 29.61 & \multirow{8}{*}{260.25} & \multirow{8}{*}{11329} & \multirow{8}{*}{26.29} \\
\hline & $\mathrm{C} 2$ & 257.18 & 11858 & 25.98 & & & \\
\hline & C3 & 273.82 & 11260 & 27.78 & & & \\
\hline & $\mathrm{C} 4$ & 308.29 & 12930 & 31.48 & & & \\
\hline & C5 & 265.95 & 11459 & 26.86 & & & \\
\hline & C6 & 274.38 & 11718 & 27.72 & & & \\
\hline & $\mathrm{C} 7$ & 274.00 & 12420 & 27.68 & & & \\
\hline & $\mathrm{C} 8$ & 276.77 & 12920 & 27.96 & & & \\
\hline \multirow{5}{*}{3070} & CL1 & 178.14 & 12512 & 18.37 & \multirow{5}{*}{172.07} & \multirow{5}{*}{11761} & \multirow{5}{*}{17.55} \\
\hline & CL2 & 180.72 & 11837 & 18.43 & & & \\
\hline & & & & & & & \\
\hline & CL3 & 230.54 & 12497 & 23.32 & & & \\
\hline & CL4 & 170.82 & 11747 & 17.41 & & & \\
\hline
\end{tabular}


La figura 14 muestra el patrón de rotura obtenido en las piezas cortas ensayadas a compresión. Se trata de la rotura por aplastamiento esperable en piezas cortas en las que no se produce flexión lateral por pandeo.

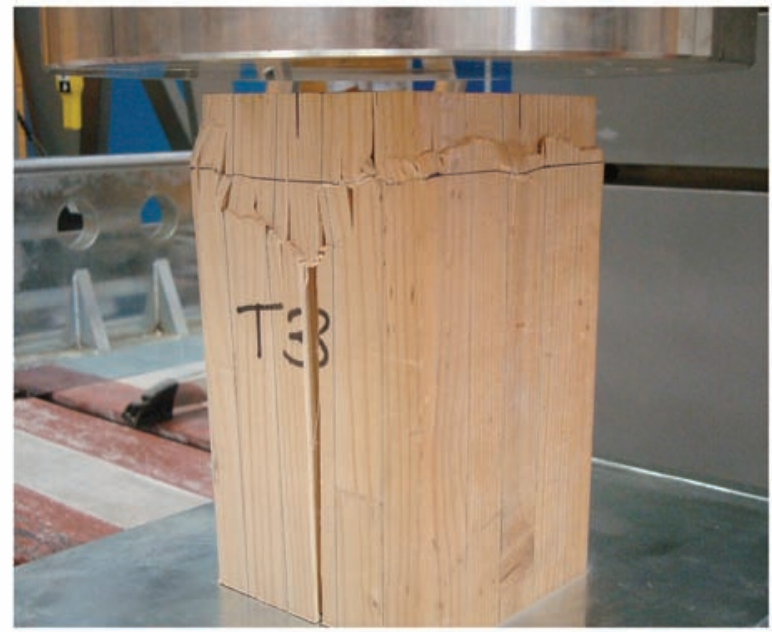

Figura 14. Forma de fallo característica de las piezas cortas sometidas a compresión.

Las figuras 15 y 16 muestran las roturas producidas en las probetas de 2050 y $3070 \mathrm{~mm}$, respectivamente. Se observa como la rotura se produce acompañada de una importante flexión lateral indicativa de que se ha alcanzado la carga crítica de pandeo. Ambas fotografías permiten comprobar la importancia del giro producido en los extremos de las barras pandeadas, lo que pone de manifiesto la eficacia del diseño con rótulas esféricas.

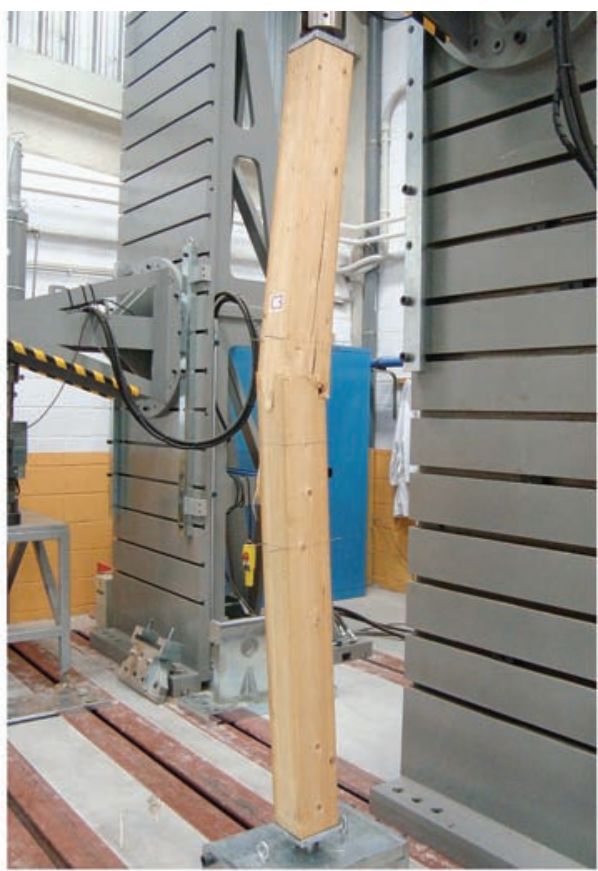

Figura 15. Forma de fallo característica de las probetas esbeltas sometidas a compresión. Ensayo probeta $\mathrm{C} 3$

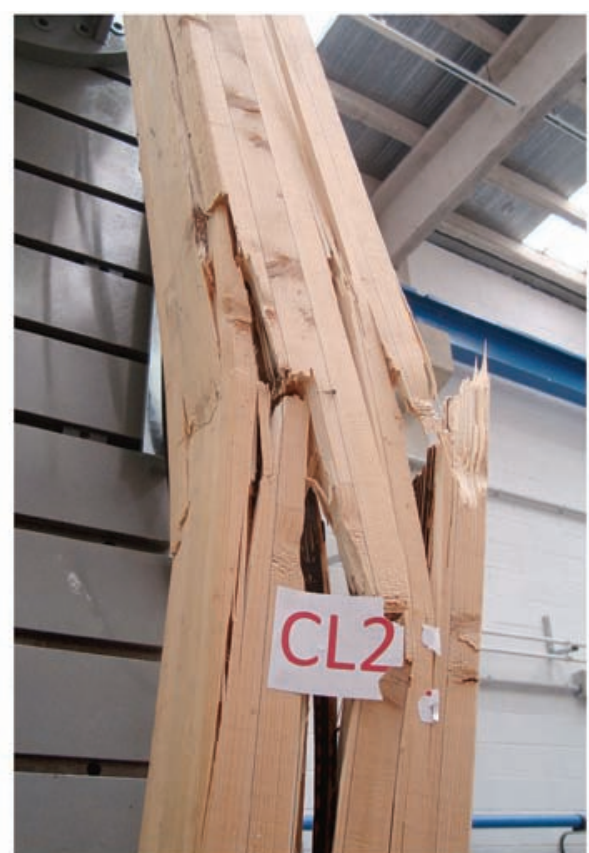

Figura 16. Forma de fallo característica de las probetas esbeltas sometidas a compresión. Ensayo probeta CL2 


\section{DISCUSIÓN}

\section{Barras sometidas a tracción}

Los resultados recogidos en la tabla 2 ponen de manifiesto que la tensión normal alcanzada en el momento del fallo correspondiente al $5 \%$ percentil de la serie ha sido de $13.48 \mathrm{~N} / \mathrm{mm}^{2}$. Este valor sube hasta $15.27 \mathrm{~N} / \mathrm{mm}^{2}$ al prescindir del resultado correspondiente a la probeta T2. Como se señaló anteriormente, dicha pieza falló para una carga claramente menor debido a un problema concreto de ejecución en la línea de encolado. Estos valores no alcanzan en todo caso la tensión característica de $19.5 \mathrm{~N} / \mathrm{mm}^{2}$ que establece la normativa UNE-EN 1194:1999 para la clase resistente de la madera empleada (GL28h). No obstante, el resultado puede considerarse muy satisfactorio pues se consigue un aprovechamiento del 69\% (78\% si se prescinde de T2) de la capacidad máxima de la pieza a tracción. Puede observarse que los resultados obtenidos para las barras reales son, además, prácticamente coincidentes con los correspondientes a los ensayos de probetas realizados con anterioridad a la construcción de la estructura. Como se recoge en la tabla 1, la tensión normal alcanzada en el momento del fallo correspondiente al $5 \%$ percentil de la serie ha sido de $14.70 \mathrm{~N} / \mathrm{mm}^{2}$, lo que representa una diferencia de tan sólo el $3.27 \%$ respecto a la tensión de rotura de las piezas reales ensayadas.

El fallo de la unión sometida a la solicitación de tracción se alcanza por la rotura a cortante de la madera en el plano inmediato a la línea de encolado. La tensión tangencial media en el momento del fallo correspondiente al $5 \%$ percentil de la serie ha sido de $1.76 \mathrm{~N} / \mathrm{mm} 2\left(2.04 \mathrm{~N} / \mathrm{mm}^{2}\right.$, si se prescinde de T2). Este valor es inferior al de $3.2 \mathrm{~N} / \mathrm{mnm}^{2}$ que establece la normativa para la clase resistente GL28h. Entendemos que ello es consecuencia de que el fallo se precipita debido a la hienda que se ha apreciado en el momento del fallo. La amplia investigación desarrollada por el equipo de investigación en el campo de las uniones encoladas (Estévez et al. 2007, Otero et al. 2008) lleva a pensar que la precipitación del fallo por hienda es consecuencia de la reducida distancia a los bordes del plano de encolado.

Por otra parte, los resultados obtenidos en términos de tensión tangencial, al prescindir de la pieza $\mathrm{T} 2$, son de nuevo prácticamente coincidentes con el resultado de los ensayos previos a la construcción de la estructura. Efectivamente la tensión tangencial media en valor característico que se obtuvo en los ensayos previos fue de $2.12 \mathrm{~N} / \mathrm{mm}^{2}$, lo que representa una diferencia del $3.77 \%$ respecto a los obtenidos a posteriori. Ello refuerza el convencimiento del problema local producido en la barra T2.

\section{Barras sometidas a compresión}

Los ensayos de piezas cortas han puesto de manifiesto que el valor obtenido de carga última a compresión de la serie correspondiente al $5 \%$ percentil $\left(36.58 \mathrm{~N} / \mathrm{mm}^{2}\right)$ supera ampliamente el correspondiente a su clasificación resistente como GL28h, que lo estable en $26.5 \mathrm{~N} / \mathrm{mm}^{2}$. También el módulo de elasticidad paralelo $5^{\circ}$ percentil obtenido en los ensayos $\left(11.16 \mathrm{kN} / \mathrm{mm}^{2}\right)$ supera el valor establecido para su clase resistente $\left(10.2 \mathrm{kN} / \mathrm{mm}^{2}\right)$.

Para analizar los resultados obtenidos en el caso de las piezas esbeltas es imprescindible la consideración de la incidencia de los fenómenos de pandeo. El Eurocódigo 5 (EN 1995-1-1) evalúa la afectación de la inestabilidad a través de un coeficiente de reducción de la tensión de cálculo. Para la obtención de dicho coeficiente la normativa ha adoptado la conocida fórmula de Rondal y Maquoi (Rondal y Maquoi 1979) que obtiene el coeficiente de reducción por pandeo $\chi$ dependiendo de la esbeltez relativa de la pieza $\lambda_{\text {rel }}$ y de un parámetro de imperfección $\eta$. 


$$
\begin{gathered}
\lambda_{\text {rel }}=\sqrt{\frac{f_{c, 0, \mathrm{k}}}{\sigma_{c, \text { crit }}}} \\
\eta=\beta \times\left(\lambda_{\text {rel }}-0.3\right) \\
\mathrm{k}=0.5 \times\left(1+\eta+\lambda_{\text {rel }}^{2}\right) \\
\chi=\frac{1}{\mathrm{k}+\sqrt{\mathrm{k}^{2}-\lambda_{\text {rel }}^{2}}}
\end{gathered}
$$

Donde $\sigma_{c, c r i t}$ es la tensión crítica de Euler, $f_{c, o, k}$ la resistencia característica a compresión paralela a la fibra y $\beta$ un factor asociado a la rectitud de la pieza para tener en cuenta la excentricidad de la carga respecto al eje de la pieza.

La fórmula de Rondal y Maquoi reproduce fielmente el comportamiento real de barras frente al pandeo a través del parámetro de imperfección $\eta$, que engloba el efecto de las imperfecciones geométricas y mecánicas, representa adecuadamente la zona de agotamiento plástico del material para esbelteces inferiores a 0.3 y permite recuperar la forma euleriana para una imperfección nula.

Para poder comparar los resultados experimentales obtenidos con los valores que predice la ecuación (4) dependiendo del parámetro $\beta$ considerado, se han representado en la figura 17 tres curvas:

- La primera es la que se obtiene aplicando la ecuación (4) considerando un parámetro $\beta$ de valor 0.1, asociado a una combadura inicial de L/500, tal y como establece el Eurocódigo 5 (UNE-EN 1995-1-1) para madera laminada encolada.

- La segunda curva corresponde a un parámetro $\beta$ de valor 0.2 , asociado a una combadura inicial de $\mathrm{L} / 300$.

- Finalmente, la tercera curva corresponde a un parámetro de valor 0.3 , no contemplado en la normativa.

En todos los casos, los cálculos se han realizado considerando los valores de tensión de compresión y módulo de elasticidad obtenidos en los ensayos. Sobre dichas gráficas se han sobrepuesto además los resultados experimentales obtenidos en forma adimensional (tensión última considerando el pandeo / tensión característica última sin consideración del pandeo). 


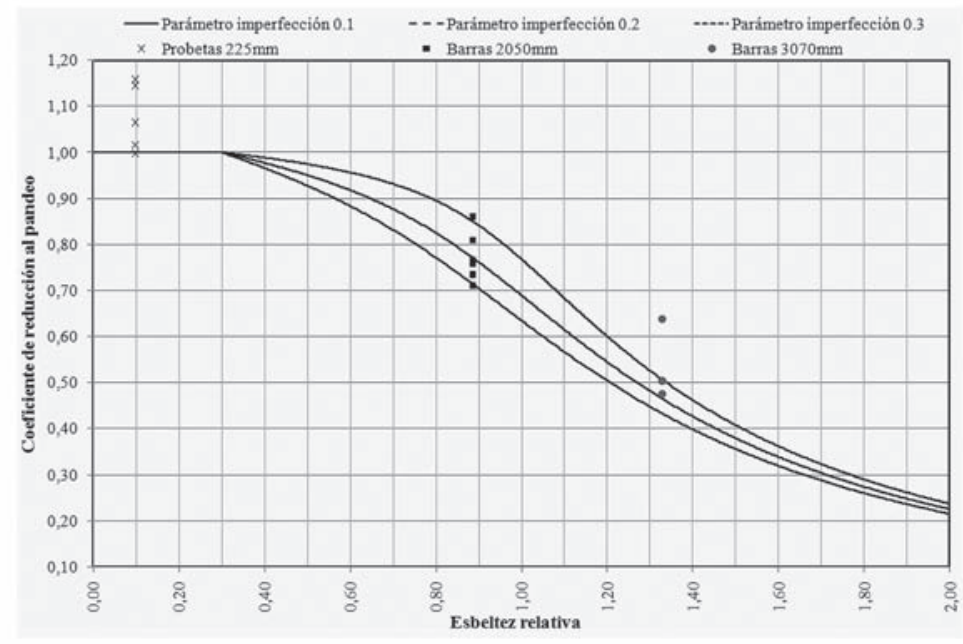

Figura 17. Resultados de los ensayos a compresión en relación con la curva europea de pandeo obtenida para diferentes parámetros de imperfección.

Los resultados ponen de manifiesto que en las barras de $2050 \mathrm{~mm}$ de longitud (esbeltez relativa de 0.89 ) las tensiones críticas en las que se ha producido el fallo por pandeo son inferiores a las predichas por el Eurocódigo 5 para la madera laminada encolada (parámetro $\beta$ de valor 0.1). Se puede observar que los resultados de la tensión última obtenida en los ensayos aconsejan considerar un parámetro $\beta$ de valor 0.3. En el caso de las barras de mayor longitud $(3070 \mathrm{~mm})$ la esbeltez relativa alcanza el valor de 1.33 y se observa que en estos casos los valores de tensión última de los resultados experimentales se sitúan entre las curvas de pandeo correspondientes al parámetro $\beta$ de valores 0.1 y 0.2 .

Estos resultados son plenamente justificables. Como es conocido, la mayor incidencia de las imperfecciones geométricas en el valor de carga crítica con la que se alcanza la inestabilidad de la forma geométrica inicial, se produce en las esbelteces más próximas a la conocida como esbeltez euleriana (valor de esbeltez en el que la tensión crítica de Euler es igual a la resistencia característica a compresión paralela), que corresponde a una esbeltez relativa de valor unidad. En ese ámbito se sitúan las barras de $2050 \mathrm{~mm}$ de longitud (esbeltez reducida de valor 0.89). El parámetro $\beta$ más desfavorable que contempla el Eurocódigo 5 es 0.2 y corresponde a una combadura de L/300. Ello quiere decir que la excentricidad de carga en la barra sería de 2050/300 $=6.8 \mathrm{~mm}$. Sin embargo, según se ha podido comprobar en las barras ensayadas, la ejecución material de la unión de extremo proyectada con una única barra roscada hace muy difícil conseguir que dicha barra esté perfectamente centrada en la sección. Como consecuencia de ello, la excentricidad total con la que se aplica la carga, considerando tanto la propia combadura de la barra como la excentricidad de extremos, supera fácilmente el valor de $6.8 \mathrm{~mm}$. Ello justifica, por tanto, que sea adecuado considerar un valor mayor para el parámetro $\beta$.

En el caso de las barras largas la situación es más favorable por dos razones: por una parte, la esbeltez de valor 1.33 está más alejada de la esbeltez euleriana, por lo que la incidencia de las imperfecciones en la carga crítica de pandeo es menor; por otra parte, la excentricidad de carga derivada de defectos en la ejecución de la unión de extremo es, en valor absoluto, igual en las barras de diferente longitud, por cuanto las secciones transversales empleadas son las mismas; sin embargo, al ser las longitudes diferentes, la imperfección relativa resultante (excentricidad de carga / luz de la pieza) en las barras más largas es menor. Ello explicaría que los resultados experimentales de las piezas más esbeltas correspondan a una curva teórica con un parámetro $\beta$ de menor valor. 


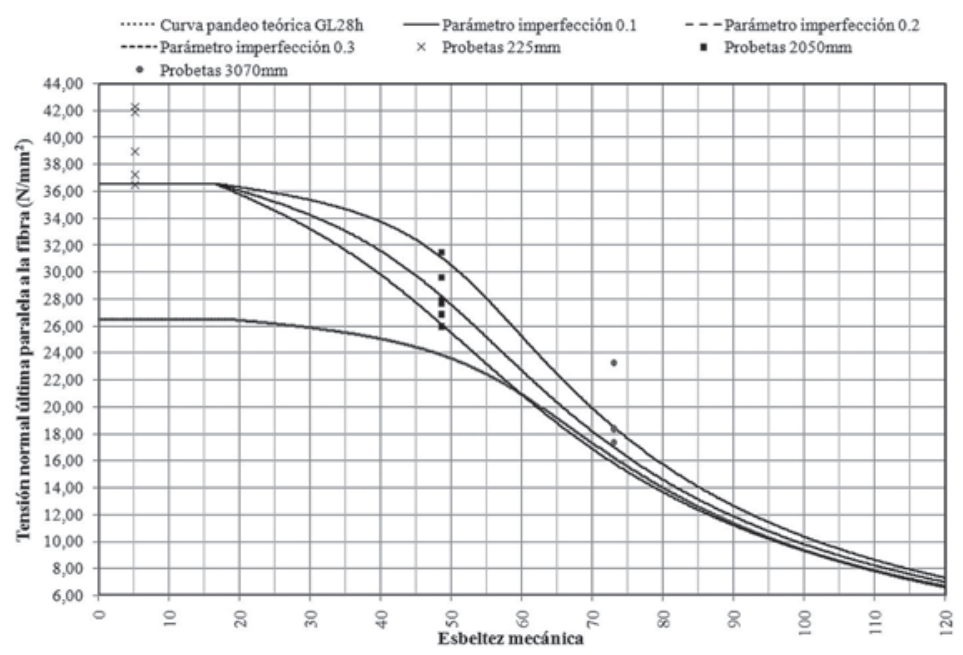

Figura 18. Resultados de los ensayos a compresión en relación con la curva europea de pandeo obtenida para diferentes parámetros de imperfección.

En la figura 18 se han representado todos los resultados anteriores pero expresados en términos de esbeltez mecánica y tensión normal última. Además se ha grafiado también la curva teórica de pandeo correspondiente a un parámetro $\beta$ de valor 0.1 , pero considerando las características mecánicas correspondientes a la clase resistente GL28h, en lugar de las obtenidas experimentalmente. Se aprecia como todos los valores de tensión alcanzados en las piezas ensayadas superan ampliamente los que corresponden a su clase resistente. También se observa cómo a medida que aumenta la longitud de las piezas y una vez que se supera la esbeltez euleriana, el factor realmente determinante en el valor de carga última es la esbeltez de las barras ensayadas y no el valor de la tensión de agotamiento del material empleado.

En todo caso, los resultados experimentales obtenidos han puesto de manifiesto la enorme eficacia del empleo de secciones tubulares de madera en piezas comprimidas esbeltas, pues se consiguen elevados radios de giro empleando una reducida cantidad de material. Ello explica que piezas biarticuladas de $3070 \mathrm{~mm}$ de longitud, como las empleadas en la malla espacial construida, con un peso total de madera de $0.132 \mathrm{kN}$ sean capaces de soportar una carga última de compresión de $172.07 \mathrm{kN}$, lo que supone un extraordinario rendimiento estructural.

Para valorar comparativamente el comportamiento de secciones tubulares en relación con las secciones macizas podemos definir el concepto de rendimiento o eficacia estructural a compresión (R) como la relación entre la carga última a compresión y el peso del elemento.

$$
\mathrm{R}=\frac{\chi \cdot \mathrm{A} \cdot \mathrm{f}_{\mathrm{c}, 0, \mathrm{k}}}{\rho \cdot \mathrm{A} \cdot \mathrm{L}}=\frac{\chi \cdot \mathrm{f}_{\mathrm{c}, 0, \mathrm{k}}}{\rho \cdot \mathrm{L}}
$$

Aplicando la expresión anterior, la relación entre el rendimiento de una sección tubular y la sección maciza queda:

$$
\frac{\mathrm{R}_{\mathrm{h}}}{\mathrm{R}_{\mathrm{m}}}=\frac{\frac{\chi_{\mathrm{h}} \cdot \mathrm{f}_{\mathrm{c}, 0, \mathrm{k}}}{\rho \cdot \mathrm{L}}}{\frac{\chi_{\mathrm{m}} \cdot \mathrm{f}_{\mathrm{c}, 0, \mathrm{k}}}{\rho \cdot \mathrm{L}}}=\frac{\chi_{\mathrm{h}}}{\chi_{\mathrm{m}}}
$$


En las expresiones anteriores $\chi$ representa el coeficiente de reducción por pandeo, $f_{c, 0, k}$ la resistencia característica a compresión paralela, $\rho$ es la densidad, $A$ el área de la sección, $L$ la luz de la pieza y los subíndices $h$ y $m$ corresponden a sección hueca y maciza, respectivamente.

La eficacia en el rendimiento a compresión de piezas tubulares comparativamente con el empleo de secciones macizas se ha representado gráficamente en la figura 19, donde puede apreciarse la ganancia de rendimiento de las secciones huecas a medida que se incrementa la luz de las piezas.

Los resultados a nivel de rendimiento justifican la adecuación de la solución de barras de madera de sección tubular para su empleo en mallas espaciales, pues permite emplear piezas de elevada dimensión, lo que se traduce en una reducción del número de nudos de la malla, que es uno de los factores de mayor repercusión en el coste final de la estructural. Por otra parte, la ejecución de las secciones tubulares es de gran sencillez, tanto si se ejecutan en madera laminada o empleando para las caras tableros de madera contralaminada o LVL. Asimismo, los resultados experimentales han demostrado la eficacia de la solución adoptada para la transmisión de cargas en los extremos de las barras por medio de la disposición de tacos de madera frondosa de elevada densidad en la que se anclan, con adhesivo epoxi, las barras roscadas de conexión a los nudos de la estructura.

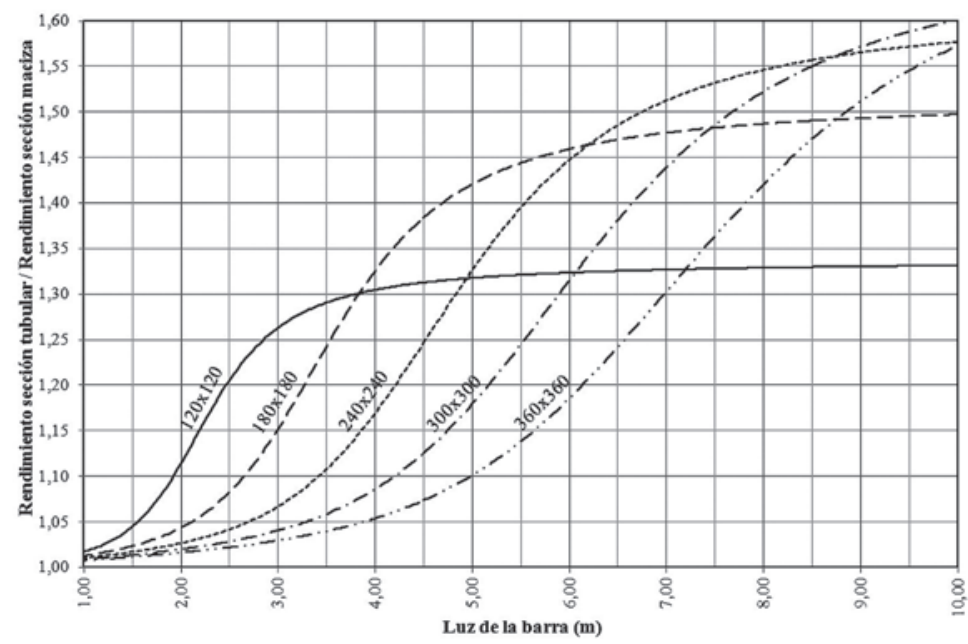

Figura 19. Rendimiento comparativo a compresión entre barras tubulares y barras de sección maciza para un espesor de pared de $25 \mathrm{~mm}$.

\section{CONCLUSIONES}

Los resultados experimentales de los ensayos de tracción han puesto de manifiesto una elevada eficacia del tipo de unión proyectado, constituido por barras roscadas ancladas con resina epoxi en tacos de madera frondosa de alta densidad que cierran los extremos de las barras tubulares. Dicha solución ha permitido aprovechar el $78 \%$ de la capacidad máxima resistente de la pieza a tracción antes de que se precipite el fallo en los extremos.

Los resultados experimentales obtenidos en los ensayos de compresión de barras esbeltas aconsejan utilizar un parámetro $\beta$ de valor 0.3 para la obtención de la capacidad última de carga, cuando la esbeltez de las barras se aproxime al valor de la esbeltez euleriana. Dicho coeficiente puede reducirse a 0.2 para esbelteces superiores a la euleriana. 
El rendimiento estructural a compresión de las piezas de madera con sección tubular en relación con el empleo de secciones macizas mejora de forma significativa al aumentar la luz de las barras. Ello convierte la solución de barras tubulares en idónea para su empleo en las estructuras con tipología de malla espacial.

\section{AGRADECIMIENTOS}

Se agradece la colaboración prestada por la Plataforma de Ingeniería de la Madera Estructural (PEMADE) de la Escuela Politécnica Superior de Lugo de la Universidad de Santiago y a su responsable D. Manuel Guaita por toda la preparación y desarrollo de los ensayos experimentales.

\section{BIBLIOGRAFÍA}

Estévez C, J.; Vázquez R, J. 2004. Spatial truss of hollow bars made of laminated timber supported by walls of reinforced masonry. Journal of the International Association for Shell Spatial Structures 45(1):13-21.

Estévez C, J.; Vázquez R, J.; Otero C, D. 2007. Experimental behaviour of threaded steel rods glued into high-density hardwood. International Journal of Adhesion and Adhesives 27(2):136-44.

Ghavami, K.; Moreira, L. 1993. Double layer bamboo space structures. Space Structures 4. Thomas Telford, London. Fourth International Conference on Space Structures 1: 573-581.

Huybers, P. 1986. Timber pole space frames. Space Structures 3. Thomas Telford, London. Third International Conference on Space Structures 2: 77-86.

Otero C, D.; Estévez C, J.; Martín G, E. 2008. Glued joints in hardwood timber. International Journal of Adhesion and Adhesives 28(8):457-463.

Rondal J.; Maquoi R. 1979. Formulations d'Ayrton-Perry pour le flambement des barres métalliques. Construction métallique 4:41-53.

Shoei Y, H. 1988. Elastic architecture. Elastic interface of wood and steel bolt. [Disponible en] $<$ http://www.jade.dti.ne.jp/shoeiyoh/Essay/ELASTIC\%20ARCHITECTURE.pdf $>$

UNE-EN 408:2011. Estructuras de madera. Madera aserrada y madera laminada encolada para uso estructural. Determinación de algunas propiedades físicas y mecánicas. Asociación Española de Normalización y Certificación (AENOR). España.

UNE-EN 1194:1999. Estructuras de madera. Madera laminada encolada. Clases resistentes y determinación de los valores característicos. Asociación Española de Normalización y Certificación (AENOR). España.

UNE-EN 1995-1-1:2006. Eurocódigo 5. Proyecto de estructuras de Madera. Parte 1-1: Reglas generales y reglas para edificación. Asociación Española de Normalización y Certificación (AENOR). España.

UNE-EN 13183-1:2002. Contenido de humedad de una pieza de madera aserrada. Parte 1: Determinación por el método de secado en estufa. Asociación Española de Normalización y Certificación (AENOR). España. 